\title{
Investigation of maternal and embryo/fetal toxicity of Ephedra viridis and Ephedra nevadensis in sheep and cattle
}

RICHARD F. KEELER

\begin{abstract}
Ephedra vtridis and Ephedra nevadensis were gavaged at high doses to pregnant livestock to determine whether there existed any maternal or embryo/fetal toxicity. $E$. viridis was teated in both sheep and cows and was toxic, and generally induced ruminal impaction, diarrhea, vomition or anorexia. E. nevadensts was tested only in sheep, but was devoid of toxicity. Neither plant induced apparent adverse effects in unborn offspring of animals gavaged. They were normal at birth.

Keywords: Ephedra spp., poisonous plants

Many plant genera contain compounds that are substituted $\beta$-phenethylamines. The phenethylamines are very active compounds biologically. Many are potent vasoconstrictors (pressor amines) and thereby greatly elevate blood pressure. In addition, they may exert a variety of physiologic effects including acceleration of heart rate, increased cardiac output, increased respiratory volume, increased central vasomotor activity, and mydriasis. They are useful medicinally because of those as well as vasoconstriction effects (Goodman and Gilman 1960). Of course, phenethylamines are considered toxic at elevated doses as is the case with all physiologically active compounds.

Some Ephedra species contain substituted $\beta$-phenethylamines.

\footnotetext{
The author is with USDA/ARS/ Poisonous Plant Research Laboratory, 1150 East 1400 North, Logan, Utah 84321.

The author expresses appreciation to Ms. Jan Knight and Mr. Glenn Patterson, BLM Hanksville, Utah, for advice on plants and collection sites; to Mr. Edwin Lambert and L.D. Balls for excellent technical assistance; and to Drs. Ward Crowe, Stan Allen, and Ross Smart for advice and necropsies.

Manuscript accepted 25 August 1988.
}

The pressor amines in Ephedra include (-)-ephedrine, (+)-pseudoephedrine, (-)-norephedrine, (-)-N-methylpseudoephedrine, and $(+)$-norpseudoephedrine, $(+)-\mathrm{N}$-methylpseudoephedrine (Brossi and Pecherer 1970). They are very close in pressor amine activity and in structure to the adrenal hormones epinephrine (adrenalin) and norepinephrine (noradrenalin) in which, contrary to the Ephedro amines, there are hydroxyl groups on the phenyl moiety. Both adrenalin and noradrenalin have been found to induce birth defects (teratogenic effects) in rabbits, rats, and chicks (Jost et al. 1969, Jost 1953, Gatling 1962, Hodach et al. 1974, Pitel and Lerman 1962).

Because of the widespread ingestion of species of the Ephedra genus by livestock on western ranges in the U.S., and because of the use of these same species in preparation of herbal teas, I investigated the toxicity and teratogenicity in sheep and cattle of 2 of the more common Great Basin species-Ephedra nevadensis S. Wats. and Ephedra viridis Coville and compared toxicoses induced to levels of suspect toxins. This report describes the results of those investigations.

\section{Materials and Methods}

\section{Plant Material}

Composite samples from several dozen Ephedra nevadensis plants were collected at an elevation of about $1,460 \mathrm{~m}$ on the Lake Bonneville benches of the Hogup and Terrace Mountains in Box Elder County, Utah, in August of 1981 (collections 81-22, $350 \mathrm{lb}$ dry weight total) and August of 1982 (collection 82-15, $470 \mathrm{lb}$ dry 
weight total). The collections included above-ground parts, excluding woody material. Composite samples from several dozen Ephedra viridis plants were collected at an elevation of about $2,130 \mathrm{~m}$ on the Eagle Bench of the Henry Mountains in Garfield County, Utah, in September of those same years (collections $81-25,540 \mathrm{lb}$ dry weight total, and 82-23, $425 \mathrm{lb}$ dry weight total). Those collections also included above-ground plant parts, excluding woody material. All 4 collections were returned to the laboratory and air dried for several days. They were then ground to a fine consistency (to pass a 15-mesh screen) and stored at ambient temperatures for later gavage. Portions subjected to chemical analysis were ground further in a Wiley Mill to pass a 40-mesh screen.

\section{Chemical Analysis}

$E$. viridis and $E$. nevadensis plant collections were subjected to tannin analysis by the Folin-Denis modification method of Burns (1963) using gallotanic acid as the standard to yield values for "tannic acid equivalents." Values were obtained at 700 rather than $725 \mathrm{~nm}$. Samples were also analyzed for ephedrine, pseudoephedrine, norephedrine, and norpseudoephedrine by gas chromatography (GC) analysis of the purified total basic fraction of the plant prepared as follows. Twenty $g$ of plant material was moistened with $20 \mathrm{ml} 2 \% \mathrm{Na}_{2} \mathrm{CO}_{3}$ and extracted for 2 days with $300 \mathrm{ml}$ benzene. The benzene was removed by filtration, pooled with an additional $100 \mathrm{ml}$ benzene wash, evaporated to $10 \mathrm{ml}$, and partitioned against $75 \mathrm{ml}$ of $1 \% \mathrm{HCl}(2 \mathrm{X})$. The separated acid phase was then neutralized to $\mathrm{pH} 10$ with $5 \% \mathrm{NH}_{4} \mathrm{OH}$ and partitioned against $100 \mathrm{ml}$ benzene $(2 \mathrm{X})$. The pooled benzene was dried and the residue taken up in ethanol for gas chromatography on an OV-17 column isothermally at $120^{\circ} \mathrm{C}$, and by temperature programming at $120^{\circ}-$ $170^{\circ} \mathrm{C}$. Standards of ephedrine, pseudoephedrine, norephedrine, and norpseudoephedrine were compared to plant extracts by GC analysis.

\section{Animal Care and Administration of Plant Materials}

Experimental animals were Hereford cows weighing an average of $410 \mathrm{~kg}$ and crossbred ewes weighing an average of $56 \mathrm{~kg}$. Ephedra plant material was administered by gavage in a water slurry at the dosage, stage of gestation, and for numbers of days indicated in Table 1. Dosages listed in Table 1 were the average dosages. There were some slight dosage adjustments $( \pm 20 \%)$ in some animals in order to arrive at a maintenance dose that elicited toxicity signs but did not result in death. Animals were provided alfalfa hay, water, and salt ad libitum.

Experimental cows were bred to Hereford bulls and ewes to Suffolk or Columbia rams with date of breeding considered as the beginning of day zero of gestation. Animals were observed for clinical signs of toxicoses throughout the gavaging period and for a few days thereafter. Offspring were examined at birth for congenital deformities. Thorough necropsies were performed on 2 of the ewes that died from toxicoses. They were selected because tissues were still reasonably fresh after death.

For measurement of blood parameters, venous blood samples from each experimental animal so tested (ewes \# 3501, 3590, 2741, $2832,3433,3436,3434,3449,3403$, and 2204 , and cows \# 3782, $3325,3753,3754$, and 3825 ) were drawn 2 days prior to gavaging, and then periodically during the period of gavaging and at 14 days after gavaging ended. Blood parameters measured singly by standard automated procedures (Abbott ABA 200 analyzer) in ewes were red blood cell count (RBC), white blood cell count (WBC), packed cell volume (PCV), hemoglobin (HGB), glutamic-oxaloactic transaminase (SGOT), glutamic pyruvic transaminase (SGPT), lactic dehydrogenase (LDH), urea nitrogen (BUN), gamma glutamyl transpeptidase (GGTP). Similar measurements were made for cows except that LDH, SGPT, and HGB were not measured.

\section{Results}

Ephedra viridis and Ephedra nevadensis differed markedly in toxicity at dosages gavaged (Table 1$)$. E. nevadensis was without toxicity whereas $E$. viridis was quite toxic at all levels gavaged. In sheep, both of the $E$. viridis collections, 81-25 and 82-23, induced ultimate clinical signs of ruminal impaction at all levels tested $(7-21 \mathrm{~g} / \mathrm{kg} /$ day) and in as little as 3 days at high doses. A few ewes had evidence of diarrhea before impaction developed. In contrast, $E$. nevadensis gavaged to sheep at $17.5 \mathrm{~g} / \mathrm{kg} /$ day for 30 days induced no clinical signs of toxicity. Only $E$. viridis was gavaged to cows and induced at doses of $4-5 \mathrm{~g} / \mathrm{kg} /$ day clinical signs of toxicity that included severe diarrhea, vomition, fecal mucus, and anorexia.

Six ewes gavaged $E$. viridis died during the course of the experiments (Table 1). Two (3419 and 3498) died from among the group gavaged collection $81-25$ at $21 \mathrm{~g} / \mathrm{kg} /$ day, one following 4 days of gavage and one following only 3 days. The other $3(3416,3103$, and 3555 ) were gavaged $21 \mathrm{~g} / \mathrm{kg}$ for only 5 days each at which time ruminal impaction became too severe to continue. In the group gavaged collection $81-25$ at $12 \mathrm{~g} / \mathrm{kg} /$ day, one died (2832) 46 days after gavage began and another died (3433) 48 days after. Necropsy established that both had impaction of rumen/abomasal contents. There were no other consistent gross pathologic signs in these 2 animals on thorough necropsy examination. The other 3 ewes (3501, 3590 and 2741) in this group, gavaged collection 81-25 at $12 \mathrm{~g} / \mathrm{kg} /$ day, survived but had signs of rumen impaction the latter $3 / 4$ of the gavaging period. In a third group, 2 ewes ( 3653 and 3678) died from rumen impaction as a result of gavaging $7 \mathrm{~g} / \mathrm{kg} /$ day, but they received a different $E$. viridis collection (82-23). No deaths were induced by $E$. nevadensis in sheep nor by $E$. viridis in cows.

There was a very marked weight loss as judged from appearance in both sheep and cows during the course of gavaging $E$. viridis as a consequence of anorexia. But careful measurements were made only on cows 3782 and 3325 . These 2 animals lost 57 and $20 \mathrm{~kg}$ respectively. Cows and sheep gavaged $E$. nevadensis, however, continued to eat normally and appeared to gain weight during the gavaging period.

There were no marked trends (e.g., clinically significant declines in RBC or WBC or elevations in the other parameters) in the 10 sheep tested in mean concentrations of any of the blood parameters measured (RBC, WBC, PCV, HGB, SGOT, SGPT, LDH, BUN, nor GGTP) during the course of gavaging either $E$. viridis or $E$. nevadensis (Table 2). Individual animal variations in these parameters during the dosing period fell within about $\pm 35 \%$ beyond the pretreatment range values except for a rise in WBC and lowering RBC in ewe \# 2204 ascribable to a respiratory infection. However, marked elevations of SGOT and LDH did occur in ewe 2832 and in LDH in ewe 3433 (Table 2) after those ewes were removed from treatment. They had been removed from gavaging at a little over $1 / 2$ the expected gavaging duration (Table 1) because of severe ruminal impaction. Values for SGOT and LDH were 128 and 1092 respectively in ewe 3433 and 181 and 1174 in ewe 2832 . These values were obtained after gavaging had ended but a few days before their deaths. SGOT and LDH values did not elevate similarly in any of the other 8 ewes tested. Values measured 14 days after termination of dosing were essentially within pretreatment ranges for surviving ewes.

There were no marked trends in the 5 cows tested in mean concentrations of any of the blood parameters measured (RBC, WBC, PCV, SGOT, BUN, nor GGTP) during the course of gavaging $E$. viridis (Table 3). Individual animal variations in those parameters during the dosing period fell within about $\pm 35 \%$ beyond the pretreatment range values except for one low BUN values of 10.2 on animal 3782 ten days after dosing began. On neither the 3 nor 24 day assays was the BUN for this animal outside the pretreatment range.

All lambs and calves born to ewes or cows gavaged either $E$. $v$ viridis or $E$. nevadensis were grossly normal and without noticeable birth deformities regardless of gestational period during which 
Table 1. Effects of Ephedra spp. On cows and ewes and their offepring.

\begin{tabular}{|c|c|c|c|c|c|c|}
\hline $\begin{array}{l}\text { Plant } \\
\text { material }\end{array}$ & $\begin{array}{l}\text { Animal } \\
\text { number }\end{array}$ & $\begin{array}{c}\text { Gestation } \\
\text { period } \\
\text { gavaged }\end{array}$ & $\begin{array}{c}\text { Daily } \\
\text { average } \\
\text { dosage }(\mathbf{g} / \mathbf{k g})\end{array}$ & Toxicity signs in the dam & Pregnancy outcome & $\begin{array}{l}\text { Birth } \\
\text { wt (kg) }\end{array}$ \\
\hline \multicolumn{7}{|c|}{$\begin{array}{l}\text { Cows gavaged } \\
E \text { viridis }\end{array}$} \\
\hline \multirow[t]{2}{*}{$81-25$} & 3782 & $10-36$ & 4.9 & $\begin{array}{l}\text { Diarrhea, vomition, } \\
\text { anorexia, fecal mucus }\end{array}$ & $\begin{array}{l}\text { Delivered normal single at } \\
\text { term }\end{array}$ & 24.5 \\
\hline & 3325 & $10-36$ & 4.9 & $\begin{array}{l}\text { Diarrhea, vomition } \\
\text { anorexia, fecal mucus }\end{array}$ & $\begin{array}{l}\text { Delivered normal single } \\
\text { at term }\end{array}$ & 27.7 \\
\hline \multirow[t]{3}{*}{$82-23$} & 3753 & $40-70$ & 3.9 & $\begin{array}{l}\text { Anorexia, vomition, } \\
\text { bloaty }\end{array}$ & $\begin{array}{l}\text { Delivered normal single } \\
\text { at term }\end{array}$ & 29.1 \\
\hline & 3754 & $40-70$ & 3.9 & $\begin{array}{l}\text { Anorexia, vomition, } \\
\text { bloaty }\end{array}$ & Non-pregnant & \\
\hline & 3825 & $40-70$ & 3.9 & $\begin{array}{l}\text { Anorexia, vomition, } \\
\text { bloaty }\end{array}$ & Non-pregnant & \\
\hline \multicolumn{7}{|c|}{$\begin{array}{l}\text { Ewes gavaged } \\
\text { E. viridis }\end{array}$} \\
\hline \multirow[t]{9}{*}{$81-25$} & 3416 & $10-15$ & 21 & Ruminal impaction & $\begin{array}{l}\text { Delivered normal twins at } \\
\text { at term }\end{array}$ & $4.5,5.0$ \\
\hline & $\begin{array}{l}3103 \\
3555\end{array}$ & $\begin{array}{l}10-15 \\
10-14\end{array}$ & $\begin{array}{l}21 \\
21\end{array}$ & $\begin{array}{l}\text { Ruminal impaction } \\
\text { Ruminal impaction (died } \\
\text { at } 14 \text { days gestation) }\end{array}$ & Non-pregnant & \\
\hline & 3419 & $10-15$ & 21 & Ruminal impaction & $\begin{array}{l}\text { Delivered normal single } \\
\text { at term }\end{array}$ & 5.9 \\
\hline & 3498 & $10-13$ & 21 & $\begin{array}{l}\text { Ruminal impaction (died } \\
\text { at } 13 \text { days gestation) }\end{array}$ & & \\
\hline & 3501 & $10-40$ & 12 & Ruminal impaction & $\begin{array}{l}\text { Delivered normal single } \\
\text { at term }\end{array}$ & 5.5 \\
\hline & 3590 & $10-40$ & 12 & Ruminal impaction & $\begin{array}{l}\text { Delivered normal twins } \\
\text { at term }\end{array}$ & $3.6,2.7$ \\
\hline & 2741 & $10-40$ & 12 & Ruminal impaction & $\begin{array}{l}\text { Delivered normal twins } \\
\text { at term }\end{array}$ & $4.5,4.1$ \\
\hline & 2832 & $10-26$ & 12 & $\begin{array}{l}\text { Ruminal impaction (died at } \\
56 \text { days gestation) }\end{array}$ & & \\
\hline & 3433 & $10-28$ & 12 & $\begin{array}{l}\text { Ruminal impaction (died at } \\
58 \text { days gestation) }\end{array}$ & & \\
\hline \multirow[t]{7}{*}{$82-23$} & 3650 & $30-60$ & 5.3 & Ruminal impaction & $\begin{array}{l}\text { Delivered normal single at } \\
\text { term }\end{array}$ & 5.9 \\
\hline & 3653 & $30-35$ & 7 & $\begin{array}{l}\text { Ruminal impaction (died } \\
\text { at } 36 \text { days gestation) }\end{array}$ & & \\
\hline & 3678 & $30-33$ & 7 & $\begin{array}{l}\text { Ruminal impaction (died } \\
\text { at } 34 \text { days gestation) }\end{array}$ & & \\
\hline & 3661 & $30-60$ & 5.3 & Ruminal impaction & $\begin{array}{l}\text { Delivered normal twins } \\
\text { at term (one had equivocal } \\
\text { forelimb bowing) }\end{array}$ & $3.2,3.2$ \\
\hline & 3656 & $30-60$ & 5.3 & Ruminal impaction & $\begin{array}{l}\text { Delivered normal twins } \\
\text { at term }\end{array}$ & $3.6,3.2$ \\
\hline & 3687 & $30-60$ & 5.3 & Ruminal impaction & $\begin{array}{l}\text { Delivered normal twins } \\
\text { at term }\end{array}$ & $4.5,5.0$ \\
\hline & 3681 & $30-60$ & 5.3 & Ruminal impaction & $\begin{array}{l}\text { Delivered normal twins } \\
\text { at term }\end{array}$ & $4.3,4.5$ \\
\hline \multicolumn{7}{|c|}{$\begin{array}{l}\text { Ewes gavaged } \\
\text { E. nevadensis }\end{array}$} \\
\hline \multirow[t]{4}{*}{$81-22$} & 3436 & $10-40$ & 17.5 & No toxic signs & $\begin{array}{l}\text { Delivered normal twins } \\
\text { at term }\end{array}$ & $4.5,5.0$ \\
\hline & $\begin{array}{l}3434 \\
3449\end{array}$ & $\begin{array}{l}10-40 \\
10-40\end{array}$ & $\begin{array}{l}17.5 \\
17.5\end{array}$ & $\begin{array}{l}\text { No toxic signs } \\
\text { No toxic signs }\end{array}$ & $\begin{array}{l}\text { Delivered normal twins } \\
\text { Delivered normal single } \\
\text { at term }\end{array}$ & $\begin{array}{l}4.5,5.5 \\
5.9\end{array}$ \\
\hline & 3403 & $10-40$ & 17.5 & No toxic signs & $\begin{array}{l}\text { Delivered normal twins } \\
\text { at term }\end{array}$ & $4.5,5.5$ \\
\hline & 2204 & $10-40$ & 17.5 & No toxic signs & $\begin{array}{l}\text { Delivered normal twins } \\
\text { at term }\end{array}$ & $5.0,5.5$ \\
\hline \multirow[t]{5}{*}{$82-15$} & 3692 & $30-60$ & 17.5 & No toxic signs & $\begin{array}{l}\text { Delivered normal twins } \\
\text { at term }\end{array}$ & $5.0,5.3$ \\
\hline & 3684 & $30-60$ & 17.5 & No toxic signs & $\begin{array}{l}\text { Delivered normal twins } \\
\text { at term }\end{array}$ & $2.7,2.7$ \\
\hline & 3662 & $30-60$ & 17.5 & No toxic signs & $\begin{array}{l}\text { Delivered normal single } \\
\text { at term }\end{array}$ & 6.4 \\
\hline & 3695 & $30-60$ & 17.5 & No toxic signs & $\begin{array}{l}\text { Delivered normal single } \\
\text { at term }\end{array}$ & 5.0 \\
\hline & 3704 & $30-60$ & 17.5 & No toxic signs & $\begin{array}{l}\text { Delivered normal twins } \\
\text { at term }\end{array}$ & $3.0,3.2$ \\
\hline
\end{tabular}


Table 2. Blood parameter means and ranges from ewes ${ }^{1}$ during gavaging of Ephedre app. and 14 days after gavaging had ended.

\begin{tabular}{|c|c|c|c|c|c|c|c|c|c|c|}
\hline & & $\begin{array}{c}\text { RBC } \\
\left(\mathrm{N} / \mathbf{m m}^{2}\right)\end{array}$ & $\begin{array}{c}\mathrm{WBC} \\
\left.\mathrm{N} / \mathrm{mm}^{2}\right)\end{array}$ & $\begin{array}{c}\text { PCV } \\
(\%)\end{array}$ & $\begin{array}{l}\text { HGB } \\
(\mathrm{g} / \mathrm{dl})\end{array}$ & $\begin{array}{c}\text { SGOT } \\
(u / 1)\end{array}$ & $\begin{array}{l}\text { SGPT } \\
(\mathrm{u} / \mathrm{l})\end{array}$ & $\begin{array}{l}\text { LDH } \\
(u / 1)\end{array}$ & $\begin{array}{c}\text { BUN } \\
(\mathrm{mg} / 100 \mathrm{cc})\end{array}$ & $\begin{array}{c}\text { GGTP } \\
(\mathrm{u} / \mathrm{l})\end{array}$ \\
\hline $\begin{array}{l}2 \text { days } \\
\text { before } \\
\text { gavaging }\end{array}$ & & $\begin{array}{c}11.0 \\
{[8.3-13.2]}\end{array}$ & $\begin{array}{c}8860 \\
{[5780-13000]}\end{array}$ & {$\left[\begin{array}{c}44 \\
{[34-46]}\end{array}\right.$} & $\begin{array}{c}15.4 \\
{[14.6-16.8]}\end{array}$ & $\begin{array}{c}83 \\
{[35-112]}\end{array}$ & $\begin{array}{c}16.9 \\
{[11.2-24.1]}\end{array}$ & $\begin{array}{c}358 \\
{[308-449]} \\
\end{array}$ & $\begin{array}{c}19.3 \\
{[14.4-25.4]}\end{array}$ & $\begin{array}{c}58.9 \\
{[44.3-83.9]} \\
\end{array}$ \\
\hline \multirow{2}{*}{$\begin{array}{l}3 \text { days } \\
\text { after } \\
\text { gavaging } \\
\text { began }\end{array}$} & E. nevadensis & $\begin{array}{c}11.2 \\
{[10.7-13.1]}\end{array}$ & $\begin{array}{c}8940 \\
{[7020-10800]}\end{array}$ & $\begin{array}{c}42 \\
{[39-43]}\end{array}$ & $\begin{array}{c}16.4 \\
{[15.7-16.9]}\end{array}$ & $\begin{array}{c}99 \\
{[82-118]}\end{array}$ & $\begin{array}{c}14.2 \\
{[12.0-17.7]}\end{array}$ & $\begin{array}{c}360 \\
{[314-403]}\end{array}$ & $\begin{array}{c}22.3 \\
{[12.9-27.1]}\end{array}$ & $\begin{array}{c}54.3 \\
{[49.6-58.7]}\end{array}$ \\
\hline & E. viridis & $\begin{array}{c}9.9 \\
{[8.6-10.6]}\end{array}$ & $\begin{array}{c}9520 \\
{[6990-12600]}\end{array}$ & $\begin{array}{c}40 \\
{[35-43]}\end{array}$ & $\begin{array}{c}15.3 \\
{[13.8-16.6]} \\
\end{array}$ & $\begin{array}{c}94 \\
{[48-124]}\end{array}$ & $\begin{array}{c}17.3 \\
{[9.1-23.9]} \\
\end{array}$ & $\begin{array}{c}405 \\
{[337-458]} \\
\end{array}$ & $\begin{array}{c}18.8 \\
{[14.0-24.3]}\end{array}$ & $\begin{array}{c}59.3 \\
{[49.6-75.5]}\end{array}$ \\
\hline \multirow{2}{*}{$\begin{array}{l}10 \text { days } \\
\text { after } \\
\text { gavaging } \\
\text { began }\end{array}$} & E. nevadensis & $\begin{array}{c}10.3 \\
{[9.3-11.6]}\end{array}$ & $\begin{array}{c}7900 \\
{[7620-8360]}\end{array}$ & $\begin{array}{c}41 \\
{[40-42]}\end{array}$ & No Data & $\begin{array}{c}73 \\
{[56-84]}\end{array}$ & $\begin{array}{c}14.5 \\
{[14.4-14.6]}\end{array}$ & $\begin{array}{c}282 \\
{[266-314]}\end{array}$ & $\begin{array}{c}19.5 \\
{[12.9-26.7]}\end{array}$ & $\begin{array}{c}54.8 \\
{[49.6-59.1]}\end{array}$ \\
\hline & E. viridis & $\begin{array}{c}8.9 \\
{[7.1-10.3]}\end{array}$ & $\begin{array}{c}7700 \\
{[4770-11000]}\end{array}$ & $\begin{array}{c}43 \\
{[36-46]}\end{array}$ & No Data & $\begin{array}{c}79 \\
{[42-109]}\end{array}$ & $\begin{array}{c}7.8 \\
{[4.4-12.2]}\end{array}$ & $\begin{array}{c}337 \\
{[283-435]} \\
\end{array}$ & $\begin{array}{c}11.2 \\
{[6.0-15.1]}\end{array}$ & $\begin{array}{c}59.2 \\
{[52.2-74.7]} \\
\end{array}$ \\
\hline \multirow{2}{*}{$\begin{array}{l}17 \text { days } \\
\text { after } \\
\text { gavaging } \\
\text { began }\end{array}$} & E. nevadensis & $\begin{array}{c}9.8 \\
{[9.5-10.5]}\end{array}$ & $\begin{array}{c}7790 \\
{[6880-9070]}\end{array}$ & $\begin{array}{c}39 \\
{[37-41]}\end{array}$ & $\begin{array}{c}15.0 \\
{[14.4-16.0]}\end{array}$ & $\begin{array}{c}75 \\
{[63-81]}\end{array}$ & $\begin{array}{c}12.0 \\
{[9.5-14.4]}\end{array}$ & $\begin{array}{c}319 \\
{[286-362]}\end{array}$ & $\begin{array}{c}18.5 \\
{[13.3-21.2]}\end{array}$ & $\begin{array}{c}58.6 \\
{[51.9-63.6]}\end{array}$ \\
\hline & E. viridis & $\begin{array}{c}9.0 \\
{[8.1-9.8]}\end{array}$ & $\begin{array}{c}7120 \\
{[7010-10300]}\end{array}$ & $\begin{array}{c}40 \\
{[35-46]} \\
\end{array}$ & $\begin{array}{c}14.4 \\
{[12.9-17.2]}\end{array}$ & $\begin{array}{c}84 \\
{[43-139]} \\
\end{array}$ & $\begin{array}{c}10.1 \\
{[11.2-12.9]}\end{array}$ & $\begin{array}{c}370 \\
{[235-309]} \\
\end{array}$ & $\begin{array}{c}13.1 \\
{[12.0-14.1]}\end{array}$ & No Data \\
\hline \multirow{2}{*}{$\begin{array}{l}24 \text { days } \\
\text { after } \\
\text { gavaging } \\
\text { began }\end{array}$} & E. nevadensis & $\begin{array}{c}8.7 \\
{[5.0-10.2]}\end{array}$ & $\begin{array}{c}8580 \\
{[6200-13800]}\end{array}$ & $\begin{array}{c}40 \\
{[37-44]}\end{array}$ & No Data & $\begin{array}{c}89 \\
{[71-112]}\end{array}$ & $\begin{array}{c}13.0 \\
{[9.9-15.8]}\end{array}$ & $\begin{array}{c}312 \\
{[252-360]}\end{array}$ & $\begin{array}{c}20.2 \\
{[17.1-22.4]}\end{array}$ & No Data \\
\hline & E. viridis & $\begin{array}{c}8.5 \\
{[7.1-10.1]}\end{array}$ & $\begin{array}{c}7860 \\
{[6880-8970]}\end{array}$ & $\begin{array}{c}38 \\
{[35-41]}\end{array}$ & No Data & No Data & $\begin{array}{c}11.3 \\
{[4.9-17.7]}\end{array}$ & $\begin{array}{c}297 \\
{[260-338]} \\
\end{array}$ & $\begin{array}{c}18.9 \\
{[17.6-20.9]}\end{array}$ & $\begin{array}{c}58.6 \\
{[45.2-65.2]}\end{array}$ \\
\hline \multirow{2}{*}{$\begin{array}{l}30 \text { days } \\
\text { after } \\
\text { gavaging } \\
\text { began }\end{array}$} & E. nevadensis & $\begin{array}{c}7.9 \\
{[3.1-9.5]}\end{array}$ & $\begin{array}{c}13900 \\
{[6360-35900]}\end{array}$ & $\begin{array}{c}37 \\
{[34-40]}\end{array}$ & $\begin{array}{c}13.9 \\
{[12.4-14.5]}\end{array}$ & $\begin{array}{c}81 \\
{[70-97]}\end{array}$ & $\begin{array}{c}13.0 \\
{[8.7-14.4]}\end{array}$ & $\begin{array}{c}335 \\
{[274-426]}\end{array}$ & $\begin{array}{c}24.5 \\
{[21.8-28.2]}\end{array}$ & No Data \\
\hline & E. viridis & $\begin{array}{c}8.5 \\
{[7.2-10.4]}\end{array}$ & $\begin{array}{c}8210 \\
{[5630-12400]}\end{array}$ & $\begin{array}{c}36 \\
{[32-42]}\end{array}$ & $\begin{array}{c}13.8 \\
{[12.4-16.0]}\end{array}$ & $\begin{array}{c}52 \\
{[42-62]} \\
\end{array}$ & $\begin{array}{c}7.6 \\
{[4.0-10.6]}\end{array}$ & $\begin{array}{c}314 \\
{[266-370]}\end{array}$ & $\begin{array}{c}18.3 \\
{[14.6-21.2]}\end{array}$ & $\begin{array}{c}62.5 \\
{[49.8-72.6]}\end{array}$ \\
\hline \multirow{2}{*}{$\begin{array}{l}14 \text { days } \\
\text { after } \\
\text { gavaging } \\
\text { ended }\end{array}$} & E. nevadensis & $\begin{array}{c}8.6 \\
{[6.1-9.9]}\end{array}$ & $\begin{array}{c}7420 \\
{[6330-9030]}\end{array}$ & $\begin{array}{c}38 \\
{[36-42]}\end{array}$ & $\begin{array}{c}14.3 \\
{[13.4-15.8]}\end{array}$ & $\begin{array}{c}65 \\
{[60-72]}\end{array}$ & $\begin{array}{c}11.0 \\
{[8.7-12.8]}\end{array}$ & $\begin{array}{c}289 \\
{[238-335]}\end{array}$ & $\begin{array}{c}27.8 \\
{[18.3-28.8]}\end{array}$ & $\begin{array}{c}54.6 \\
{[47.3-62.9]}\end{array}$ \\
\hline & E. viridis & $\begin{array}{c}9.3 \\
{[7.5-10.2]}\end{array}$ & $\begin{array}{c}8260 \\
{[6550-10900]}\end{array}$ & $\begin{array}{c}40 \\
{[33-47]}\end{array}$ & & $\begin{array}{c}91 \\
{[40-181]}\end{array}$ & $\begin{array}{c}11.9 \\
{[8.2-15.2]}\end{array}$ & $\begin{array}{c}639 \\
{[271-1170]}\end{array}$ & $\begin{array}{c}18.9 \\
{[10.8-26.4]}\end{array}$ & $\begin{array}{c}70.8 \\
{[48.2-110]} \\
\end{array}$ \\
\hline
\end{tabular}

There were 5 ewes gavaged $E$. nevadensis (\# 3436, 3434, 3449, 3403 \& 2204), and S ewes gavaged $E$. viridis (\# 3501, 3590, 2741, 2832 \& 3433). However, 2 on $E$. viridis (\# 2832 \& 3433) were not gavaged beyond 16 and 18 days respectively. $E$. viridis data for the 24 and 30 day periods does not include those 2 ewes. Data from all ewes are included in the 14 days after gavaging period, however.

Table 3. Blood parameter means and ranges from cows' during gavaging of Ephedra viridis.

\begin{tabular}{|c|c|c|c|c|c|c|}
\hline & $\begin{array}{c}\mathbf{R B C} \\
\left(\mathrm{N} / \mathrm{mm}^{2}\right)\end{array}$ & $\begin{array}{c}\mathrm{WBC} \\
\left(\mathrm{N} / \mathrm{mm}^{2}\right)\end{array}$ & $\begin{array}{l}\text { PCV } \\
(\%)\end{array}$ & $\begin{array}{c}\text { SGOT } \\
(u / 1)\end{array}$ & $\begin{array}{c}\text { BUN } \\
(\mathrm{mg} / 100 \mathrm{cc})\end{array}$ & $\begin{array}{c}\text { GGPT } \\
(u / 1)\end{array}$ \\
\hline $\begin{array}{l}2 \text { days before } \\
\text { gavaging }\end{array}$ & $\begin{array}{c}7.3 \\
{[6.4-8.1]} \\
\end{array}$ & $\begin{array}{c}6470 \\
{[5700-6970]}\end{array}$ & $\begin{array}{c}42 \\
{[35-50]} \\
\end{array}$ & $\begin{array}{c}71 \\
{[55-91]}\end{array}$ & $\begin{array}{c}20.5 \\
{[17.2-23.0]} \\
\end{array}$ & $\begin{array}{c}16.1 \\
{[11.0-27.9]} \\
\end{array}$ \\
\hline $\begin{array}{l}3 \text { days after } \\
\text { gavaging began }\end{array}$ & $\begin{array}{c}7.4 \\
{[7.0-7.7]}\end{array}$ & $\begin{array}{c}7080 \\
{[6580-7840]}\end{array}$ & $\begin{array}{c}42 \\
{[37-50]}\end{array}$ & $\begin{array}{c}74 \\
{[61-95]}\end{array}$ & $\begin{array}{c}21.5 \\
{[15.9-25.7]}\end{array}$ & $\begin{array}{c}16.4 \\
{[12.3-25.4]}\end{array}$ \\
\hline $\begin{array}{l}10 \text { days after } \\
\text { gavaging began }\end{array}$ & $\begin{array}{c}7.7 \\
{[7.3-7.9]}\end{array}$ & $\begin{array}{c}7810 \\
{[7120-8690]}\end{array}$ & $\begin{array}{c}43 \\
{[39-48]}\end{array}$ & $\begin{array}{c}78 \\
{[45-93]} \\
\end{array}$ & $\begin{array}{c}17.1 \\
{[10.2-24.2]}\end{array}$ & $\begin{array}{c}14.8 \\
12.3-19.8] \\
\end{array}$ \\
\hline $\begin{array}{l}24 \text { days after } \\
\text { gavaging began }\end{array}$ & {$[6.7-8.9]$} & $\begin{array}{c}6710 \\
{[5510-8240]}\end{array}$ & $\begin{array}{c}44 \\
{[37-52]}\end{array}$ & $\begin{array}{c}66 \\
{[46-79]}\end{array}$ & $\begin{array}{c}19.8 \\
{[16.0-24.6]}\end{array}$ & $\begin{array}{c}14.3 \\
{[12.6-17.0]}\end{array}$ \\
\hline
\end{tabular}

IThese data are from 5 cows, 2 of which (\# 3782 \& 3325) were gavaged $E$. viridis collection $81-25$ and 3 of which (\# 3753, 3754 \& 3825] were gavaged $E$. viridis collection 82-23.

the animals were gavaged. Neither were there any trends in birth weight (Table 1) nor thriftiness in experimental offspring.

A comparison of the "tannic acid equivalent" values for $E$. nevadensis and $E$. viridis showed marked differences, although both were very high. $E$. nevadensis and $E$. viridis averaged $7.77 \%$ and $28.5 \%$ "tannic acid equivalents," respectively, on a plant dry weight basis. GC analysis of plant samples for ephedrine, pseudoephedrine, norephedrine, and norpseudoephedrine revealed that neither species had measurable levels of these components (experimental method threshold limits $=0.2 \mu \mathrm{g} /$ injected sample). Based on that detection limit, neither plant could have had more than $1 \times 10^{-5}$ percent of any of the 4 compounds.

\section{Discussion}

The Ephedra spp. of the Great Basin are generally considered by ranchers as suitable forage, particularly, for sheep. $E$. viridis, $E$. nevadensis and other species found in the region generally show evidence of having been grazed. In fact, $E$. nevadensis is usually grazed heavily. These rancher observations are supported by the literature. For example, Welsh et al. (1987) report that $E$. nevadensis 
".... is consistently hed ged back by sheep, to whom it is a valuable source of browse," while $E$. viridis "..... is not so severely hedged by browsing animals as some of the other species, but is still of considerable importance."

Teas brewed from branchlets of several of the Ephedra species including $E$. nevadensis and $E$. viridis have been popular for medicinal and recreational purposes for centuries (Dobelis 1986, Harrington 1967). These teas were utilized by early white settlers in the Great Basin and were called by a variety of names including Brigham or Mormon tea. American Indians of the region, even earlier, used such teas or ground seed or plant material for colds, fever, or diuretics, as decongestants and for several other purposes (Dobelis 1986). But unlike some of the Ephedras from other parts of the world, the compound ephedrine and other B-phenethylamines were not found in $E$. viridis or $E$. nevadensis by investigators $50-60$ years ago (Terry 1927, Read and Feng 1927, Nielsen and McCausland 1928, and Nielsen et al. 1927). This is somewhat surprising in view of the described medicinal uses. These uses suggest the presence of one or more of the group even if at lower concentration. Tannins were reported to be present at high levels in one of those early studies (Terry 1927).

The toxicity signs elicited by $E$. viridis in sheep and cattle in the studies reported here were consistent with presence of a high tannin content. Ruminal impaction, diarrhea, anorexia and fecal mucus are found in livestock from ingestion of other plants of high tannin content. These signs are common, for example, with the various oaks (James et al. 1980, Panciera 1978, Kingsbury 1964). Certain toxicity signs in livestock characteristic of phenethylamine compounds were not observed. There was no ataxia or prostration as in guajillo wobbles from the $\mathrm{N}$-methyl- $\beta$-phenethylamine from $\mathrm{Aca}$ cia berlandieri Benth. (Kingsbury 1964). There was no cardiovascular collapse as with $\beta$-phenethylamines from Phorodendron or Viscum genera (Kingsbury 1964). However, the characteristic action of ephedrine on smooth musculature could readily account for the ruminal impaction wherein gastrointestinal tract musculature is relaxed thereby slowing peristalsis (Goodman and Gilman 1960). Van Liere et al. (1936) showed stomach emptying time to be considerably delayed upon oral administration of ephedrine in man. Even if $\beta$-phenethylamine compounds were present in the plants at the detection limit of the methods $\left(1 \times 10^{-6} \%\right)$, the resultant sheep or cow doses would not have exceeded 1/25th the therapeutic human oral dose of ephedrine for asthma relief $(50 \mathrm{mg}$ orally). The ruminal impaction in sheep from Ephedra gavage is most likely due to tannins rather than $\beta$-phenethylamine compounds based on the analytical results reported here. And this is surprising since there were no marked elevations (doubling or more) in BUN levels in our experimental animals expected from tannin toxicosis as are characteristic of oak poisoning due to the resultant renal damage from contained tannins (Panciera 1978, Householder and Dollahite 1963). Other deviations in individual or mean values for blood parameters measured during the course of gavaging Ephedra to sheep or cows are considered to be of no clinical significance. The elevations in SGOT and LDH in ewes 3433 and 2832 after removal from treatment, and prior to death, were clinically significant and represent active lesion processes, but it remains to be seen whether they represent primary or secondary effects from Ephedra toxicity.

The lack of congenital deformities in offspring from sheep or cows gavaged very high dosages of $E$. viridis or in offspring from sheep gavaged high dosages of $E$. nevadensis suggests a degree of safety exists for concepti of pregnant animals ingesting these plants. This despite severe toxicity from $E$. viridis in cows and ewes. Further experiments at lower dosages for extended gestational periods with additional animals seem unwarranted, unless field reports at some future time suggest otherwise.

On the basis of these experiments, Ephedra nevadensis seems to be perfectly safe for grazing livestock since it induces neither toxicity in ewes or cows gavaged the plant, nor congenital deformities in lambs. $E$. viridis, on the other hand, has obvious potential to cause toxicosis in grazing sheep or cattle but not birth defects in offspring. Ranchers and land management personnel should be aware that toxicosis masquerading superficially as oak brush poisoning may actually be due to Ephedra viridis. Amounts used by humans for brewing tea are orders of magnitude lower than the levels gavaged these experimental animals. It seems unlikely that serious short-term toxicoses would result therefrom.

\section{References}

Brosal, A., and B. Pecherer. 1970. Alkaloids containing a simple aromatic moiety. p. 24. In: S. W. Pelletier (Ed.). Chemistry of the Alkaloids. Van Nostrand Reinhold, New York.

Burns, R.E. 1963. Method of tannin analysis for forage crop evaluation. Univ. Ga. Agr. Exp. Sta. Tech. Bull. N.S. 32.

Dobeliw, I.N. 1986. Magic and medicine of plants. Readers Digest Assoc. Pleasantville, N.Y. p. 254.

Gatling, R.R. 1962. The effect of sympathomimetic agents on the chick embryo. Amer. J. Pathol. 40:113-127.

Goodman, L.S., and A. Gilman. 1960. The pharmacological basis of therapeutics. The MacMillan Co., New York.

Harrington, H.D. 1967. Edible native plants of the Rocky Mountains. Univ. New Mexico, Press, Albuquerque.

Hodach, R.J., E.F. Gilbert, and J.F. Fallon. 1974. Aortic arch anomalies associated with administration of epinephrine in chick embryos. Teratology 9:203-210.

Householder, G.T., and J.W. Dollahlte. 1963. Some clinical biochemical changes in blood serum of calves fed Quercus havardi. Southwest Vet. 16:107.

James, L.F., R.F. Keeler, A.E. Johnson, M.C. Williams, E.H. Cronin, and J.D. Oleen. 1980. Plants poisonous to livestock in the Western States. USDA Agr. Info. Bull. 415.

Jost, A. 1953. Degenerescence des extremities du foetus de rat provoquee par i'adrenaline. C.R. Acad. Sci. (Paris) 236:1510-1512.

Jost, A., J. Rom, and M. Cowitat. 1969. Congenital amputations determined by the br gene and those induced by adrenalin injection in the rabbit fetus. p. 187-199. In: Swinyard, C.A. (Ed.). Limb Development and Deformity: Problems of Evaluation and Rehabilitation. Springfield: C.C. Thomas.

Kingabury, J.M. 1964. Poisonous plants of the United States and Canada. Prentice Hall, Inc. Englewood Cliffs, N.J. p. 304, 385. 444.

Nielsen, C., and H. McCausland. 1928. The occurrence and alkaloidal content of various Ephedra species. J. Amer. Pharm. Assoc. 17:427-430.

Nielsen, C., H. MeCaualand, and H.C. Spruth. 1927. The occurrence and alkaloidal content of various Ephedra species. J. Amer. Pharm. Assoc. 16:288-294.

Panciera, R.J. 1978. Oak poisoning in cattle. p. 499-506. In: R.F. Keeler, K.R. Van Kampen and L.F. James (Eds.). Effects of Poisonous Plants on Livestock. Academic Press, New York.

Pitel, M., and S. Lerman. 1962. Studies on the fetal rat lens. Effects of intrauterine adrenalin and noradrenalin. Invest. Ophthalmol. 1:406-412.

Read, B.E., and C.T. Fens. 1927. The alleged ephedrine action of two California species of Ephedra. Proc. Soc. Exptl. Biol. Med. 24:819-821.

Terry, R.E. 1927. A study of Ephedra nevadensis. J. Amer. Pharm. Assoc. 16:397-407.

Van Llere, E.J., D.H. Lough, and C.K. Sleeth. 1936. The effect of ephedrine on the emptying of the human stomach. J. Amer. Med. Assoc. 106:535-536.

Welch, S.L., N.D. Atwood, S. Goodrich, and L.C. Higrins. 1987. A Utah flora (Ephedra Family). Great Basin Natur. Memoirs. 9:28-29. 